\title{
Two-dimensional photonic band-gap structures as quasi-metals
}

\author{
P. J. Roberts \\ Defence Research Agency, St. Andrews Road, Malvern WR14 3PS, UK \\ T. A. Birks and P. St. J. Russell \\ Optoelectronics Research Centre, University of Southampton, Southampton SO17 1BJ, UK \\ T. J. Shepherd \\ Defence Research Agency, St. Andrews Road, Malvern WR14 3PS, UK \\ D. M. Atkin \\ Optoelectronics Research Centre, University of Southampton, Southampton SO17 1BJ, UK
}

Received October 16, 1995

\begin{abstract}
By considering waves that propagate out of the transverse plane, we show that common high-index materials (e.g., GaAs) with a two-dimensional array of air holes can act in some ways as three-dimensional photonic band-gap structures. In particular, we describe a dielectric quasi-metal that reflects all propagating light incident from free space.
\end{abstract}

Certain three-dimensional photonic crystals, artificial dielectric materials with refractive indices that vary periodically in three dimensions, have been shown to exhibit photonic band gaps (PBG's). These are ranges of optical frequency where the density of states is zero; light of such a frequency that attempts to enter the material is reflected. ${ }^{1}$ A full PBG requires an appropriate crystal structure and a large index variation within the unit cell. The task of fabricating a threedimensional photonic crystal at optical frequencies is daunting, owing to the smallness of the unit cell (approximately half a wavelength across) and the need for a sizable volume of material. This has led many workers to explore two-dimensional (2D) photonic crystals that are invariant along a longitudinal axis but periodic in the transverse plane. ${ }^{2-4}$ 2D PBG's have been reported for propagation restricted to the plane, but this restriction is artificial because in reality light is usually free to propagate in directions out of the plane, with nonzero values for the longitudinal wavevector component. Although these out-of-plane waves are rarely considered, ${ }^{2,4}$ a full understanding of the properties of a real $2 \mathrm{D}$ photonic crystal must take them into account.

In this Letter we describe a numerical study of a dielectric medium with a regular array of air holes. Although this structure has been described elsewhere, we depart from earlier studies by addressing, in particular, whether light can propagate across the structure for nonzero values of the longitudinal wave-vector component $\beta$. 2D band gaps (generalizations of the much explored in-plane band gaps but for fixed nonzero $\beta$ ) exist if such propagation is forbidden. For an experimentally attainable refractive-index contrast, we show that propagation can be forbidden for all polarization states at all values of $\beta$ permitted by incidence from vacuum at a parallel interface. The result is a dielectric quasi-metal with many potential applications.
The geometry considered comprises a hexagonal arrangement of parallel circular cylindrical rods of low index surrounded by a medium of high index (see the inset in Fig. 2 below). For generality, a normalized propagation constant $\beta \Lambda$ and a normalized frequency $k \Lambda$ are defined, where $\Lambda$ is the center spacing of adjacent rods and $k=\omega / c$ is the vacuum wave constant.

Two numerical methods have been employed in this study. The first is the $k$-space or plane-wave expansion method, ${ }^{5}$ based on a Fourier expansion of both the periodic index distribution and the field. The resulting infinite set of algebraic equations is truncated to finite size before eigenvalues are found. In the second, real-space, method, ${ }^{6}$ the field and index distributions within the unit cell are discretized on a grid of points. These points are grouped into subcells, within which the fields are related by transfer matrices. The subcells are small enough to preclude numerical instabilities caused by exponentially growing modes. The fields of adjacent subcells are then related by a numerically stable scattering matrix. For hexagonal symmetry, the field and index distributions are discretized along nonorthogonal axes, corresponding to the primitive lattice vectors of the underlying hexagonal Bravais lattice.

In both analyses, all possible waves with given $\omega$ and $\beta$ are sought. If none is found with a real transverse wave-vector component, this $\omega, \beta$ is in a $2 \mathrm{D}$ PBG. The calculation thus implicitly considers at once all possible polarizations and transverse directions of propagation. Also, the results are equally applicable for any orientation of the structure.

The results for a GaAs host (refractive index 3.639), with holes of diameter such that the air filling fraction is $80 \%$, are given in Fig. 1(a). The shaded region is the first 2D PBG, indicating states that are forbidden in the photonic crystal. The line $\beta=k$ marks the 

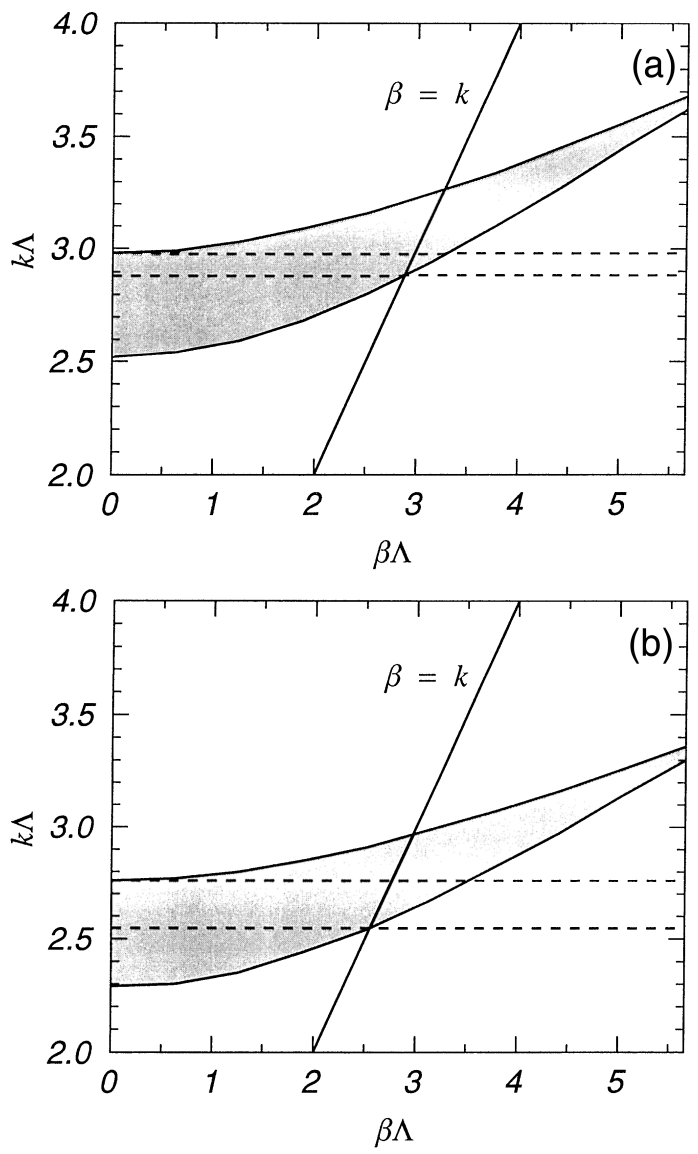

Fig. 1. First 2D PBG (shaded) of (a) GaAs/air and (b) $\mathrm{Ge} /$ air photonic crystals described in the text. States to the right of the line $\beta=k$ are forbidden in air. The dashed lines therefore mark a frequency range for which no propagating waves are excited within the photonic crystal, for propagating waves of any direction or polarization state incident from air upon a plane-parallel interface.

limit of waves that can propagate in free space. Notice that, within a narrow band of frequencies, no light can propagate in the structure with any $\beta$ that is allowed in free space. However, $\beta$ must be matched at a plane interface that is parallel to the rods. This implies that a wave in air with any angle of incidence and any polarization state will be completely reflected at such a boundary. The structure is acting as a perfect metal-a quasi-metal-despite having tworather than three-dimensional periodicity.

There is a frequency range of all-angle reflection only if the index ratio is greater than $\sim 3.3$, even after optimization with respect to the filling fraction. This range is thus fairly narrow for GaAs/air systems [ $3.5 \%$ of the center frequency; Fig. 1(a)] and for $\mathrm{Si} /$ air systems (index ratio 3.505 at $1.3 \mu \mathrm{m}$ ), but it does widen quickly with increasing index ratio. Figure 1(b) shows the band gap for an otherwise identical system with air holes in Ge (index ratio 4.02 at $3 \mu \mathrm{m}$ ), where the window of all-angle reflection extends to almost one half of the width of the in-plane $(\beta=0) \mathrm{PBG}-\sim 8 \%$ of the center frequency.

There was good agreement between the two numerical methods. For example, the results for the GaAs/air system differed by a few percent for both the band-gap plots and the refractive-index contrast at cutoff. The convergence with the number of grid points employed in the real-space method was, however, faster than the convergence with the number of included plane waves in the $k$-space method. The results from the $k$-space method approached the converged results from the real-space method slowly, as the number of included basis waves was increased. In contrast, a grid of $24 \times 24$ points was sufficient to yield accurate convergence with the real-space method, and we used it to obtain Fig. 1. The dispersion surfaces obtained by this method show almost perfect hexagonal symmetry.

Figure 1 identifies the frequencies for which no propagating waves are excited in a photonic crystal by a wave incident from free space onto a parallel surface. However, light could still tunnel through a finite slab via evanescent waves. To assess how many layers of the dielectric crystal are necessary to achieve high reflection, we studied the transmission intensity of an incident plane wave as a function of the number of layers, angle of incidence, and polarization. The attenuation per layer is generally greater far from the band edges, as expected. Figure 2 shows the total transmitted intensity maximized over all angles of incidence and incident polarization states for 10 layers of the system of Fig. 1(a). The intensity is plotted as a function of frequency within the range of all-angle reflection. The surfaces of the crystal are also indicated, though there is little dependence on the choice of truncation surface. The attenuation is greater than $99.9 \%$ over most of the range.

These results show that is feasible to construct potentially useful PBG structures by using only 2D photonic crystals. One can imagine dielectric waveguides for millimeter-wave or optical applications in which complete lateral confinement within a hollow tubular structural defect is provided by a $2 \mathrm{D}$ photonic crystal. The waveguide could support strictly one mode filling the guide (although there may be surface-guided states on the rim and high- $\beta$ free modes in the cladding, with evanescent tails extending into the guide). This structure would have applications in low-threshold lasers, with spontaneous emission largely captured in the guided mode only. In the millimeter-wave case the structure could perhaps be extruded from a drilled

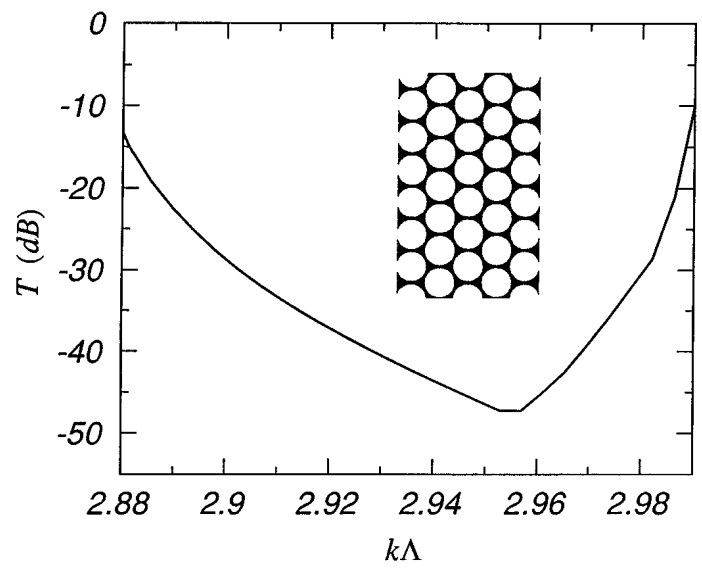

Fig. 2. Maximum total transmitted intensity $T$, in decibels, through 10 layers of the structure of Fig. 1(a). The truncation surfaces are shown in the inset. 
template and the resulting material used as a highly reflecting substrate in antenna design. At optical frequencies the quasi-metal offers an alternative to the common multilayer dielectric mirror, with designable bandwidths and wavelengths of reflection, operating at all angles of incidence and for all polarization states.

It is interesting to note that one-dimensional multilayer structures can also act as quasi-metals. However, these are more limited because light is confined in one direction only. For example, a one-dimensional structure cannot yield the hollow tubular waveguide described above.

In conclusion, it is possible to design a $2 \mathrm{D}$ photonic crystal that completely blocks the propagation of light for all directions of incidence from free space and for all states of polarization. Such a structure can be made from GaAs and air or from Si and air and would be useful in a wide range of applications.
The Optoelectronics Research Centre is an Engineering and Physical Sciences Research Council Interdisciplinary Research Centre. This work was funded partly by Defence Research Agency Malvern.

\section{References}

1. E. Yablonovitch, J. Opt. Soc. Am. B 10, 283 (1993).

2. R. D. Meade, K. D. Brommer, A. M. Rappe, and J. D. Joannopoulos, Appl. Phys. Lett. 61, 495 (1992).

3. P. R. Villeneuve and M. Piché, Phys. Rev. B 46, 4969 (1992).

4. A. A. Maradudin and A. R. McGurn, J. Mod. Opt. 41, 275 (1994).

5. K. M. Ho, C. T. Chan, and C. M. Soukoulis, Phys. Rev. Lett. 65, 3152 (1990).

6. J. B. Pendry, J. Mod. Opt. 41, 209 (1994). 